Pedro Mogorrón Huerta

\title{
Importancia (numérica) de las variantes diatópicas españolas y su tratamiento en los diccionarios ${ }^{1}$
}

\begin{abstract}
This paper describes the work done by the research team FRASYTRAM (Fraseología Y Traducción Multilingüe) at the University of Alicante, which is creating a database specialised in fixed verbal constructions (FVC) aiming at being as exhaustive as possible. This database is 29000 records rich. 9000 of them are related to FVC from different Latin American countries and 20900 have been collected from Spanish dictionaries.
\end{abstract}

The Spanish dictionaries used for creating the database only contain 1000 diatopic FVC coming from Latin America, even if two of them (Diccionario de la Real Academia Española de la Lengua and Diccionario de Uso del Español de María Moliner) are considered reference works in Spain and Latin America.

We have studied Latin American dictionaries and have identified 8100 Latin American FVC that are not registered in Spanish dictionaries. This number should increase as we have not yet finished the stage of extraction. These FVC are analysed in order to study how they are treated in Spanish and Latin American dictionaries.

In this paper we analyse parasynonyms related to „llover mucho, con fuerza, intensamente“ identified in the Spanish and Latin American dictionaries and on the Internet. It is a frequent atmospheric phenomenon in Spain and Latin American countries. In this sense different expressions should be used to describe this concept. In fact, there exist different expressions used in Spain, but are they used in Latin American countries or are they registered in Latin American dictionaries? This paper also aims at identifying expressions which are used in Latin American countries.

We have classified the FVC in four groups:

- Spanish FVC registered in Spanish dictionaries:

- [caer, llover] capuchinos de bronce (DDFEJC); [caer, llover] chuzos (de punta) (DUE); [descargar, desgajarse] [el cielo, las nubes] (DUE); llover a mares (DUE); llover a cántaros (DUE); llover a jarros (DUE); llover a mares (DUE); llover más que [cuando, el día que] enterraron [a Zafra, al Zafra] (EPM).

\footnotetext{
1 Este artículo forma parte de las investigaciones llevadas a cabo para realizar el proyecto de Investigación FFI2011-24310 «Estudio lingüístico, diatópico y traductológico de las construcciones verbales fijas más usuales en español» financiado por el Micinn.
} 
- Spanish FVC identified on the Internet:

- en Navarra y Guipuzcoa, está jareando; en Santiago de Compostela, llover a barriles; en Canarias llover más que cuando se casó Abraham (DEREC); llover más que el año que el barranco se llevó a la puerca (DEREC), etc.

- Latin American FVC registered in Latin American dictionaries:

- [caer, llover] burros aparejados (DDAmer, República Dominicana); caer sapos de punta (DFHA, Argentina); caer sapos panza arriba (DFHA; Argentina); caer soretes de punta (DFHA; Argentina); llover a baldazos (GDHA; Norte de Argentina); llover a baldes (GDHA; Norte de Argentina); estar cayendo [bigornias, pingüinos] de punta (GDHA; Argentina).

- Latin American FVC identified on the internet:

- Abrirse el cielo (Puerto Rico); caer raíles de punta (internet, Cuba); estar cayendo el cielo (internet, Venezuela); estar cayendo un palo/coñazo de agua (internet, Venezuela); estar lloviendo pescados y hasta ballenas (Internet, Guatemala); llover de abajo para arriba (Argentina, Costa Rica); llover sapos y culebras (internet, El Salvador).

The results show that dictionaries do not include most of the FVC identified in our research. Furthermore, Latin American dictionaries include idiosyncratic expressions but they do not include standard expressions. This can lead to some kind of misunderstanding. Further research is required to provide a group of phraseological units that is common to the majority of Spanish speaking countries.

Keywords: phraseological units; dictionaries; diatopic variation

Correspondence address: pedro.mogorron@ua.es

\section{Introducción}

El grupo de investigación FRASYTRAM (Fraseología y Traducción multilingüe) de la Universidad de Alicante está elaborando desde 2005 una base de datos multilingüe (BD), de Construcciones Verbales Fijas (CVF). El punto de partida de este proyecto surge al observar que muchas de las expresiones fijas que hemos intentado encontrar en diccionarios, ya sean monolingües (para obtener información acerca del significado y el uso de esas expresiones), ya sean bilingües (para encontrar la traducción ofrecida) no aparecen en estas obras. En efecto, aunque el conjunto de unidades fraseológicas seleccionadas y presentes, tanto en los diccionarios monolingües como bilingües o fraseológicos, constituye una parte importante del sistema fraseológico, esa presencia se ha plasmado generalmente 
en la incorporación de cierto número de unidades, a menudo escaso, siguiendo un proceso de selección y descripción únicamente alfabético fruto de la propia intuición y competencia lingüística del o de los lexicógrafos encargados de la elaboración de la obra y además sin ningún criterio de clasificación exhaustivo Una vez observada esa deficiencia, decidimos intentar elaborar un diccionario bajo la forma de una base de datos para incorporar el mayor número de expresiones posibles. Ante la enorme variedad de tipos de unidades fraseológicas (UF) presentes en la lengua (ver Corpas 1996; Ruiz Gurillo 1997; García-Page 2008; Zuluaga 1980; etc.), hemos integrado dentro de las CVF las siguientes expresiones fijas:

1. locuciones verbales: coger (alguien) el toro por los cuernos, (DUE); ${ }^{2}$ desnudar (alguien) un santo para vestir a otro, (DUE); hacerse (alguien) el sueco, (DUE); etc.

2. colocaciones verbales: guiñar (alguien) un ojo, (DUE); formular (alguien) una pregunta, (DUE); conciliar (alguien) el sueño, (DUE); etc.

3. verbos soportes: dar (alguien) un paseo, DUE, tomar (alguien) el aire (DUE); etc.

4. construcciones verbales comparativas: dormir (alguien) como un tronco, (DUE); estar (alguien) como pez en el agua, (DUE); etc.

5. las construcciones $\mathrm{V}+$ loc adverbial que tienen un paradigma cerrado con uno varios verbos formando una combinación de uso frecuente o de carácter preferente: llover a cántaros, (DUE); pagar (alguien) (algo) a tocateja, (DUE); conocer (alguien) (a alguien) de vista, (DUE); etc.

\section{Elaboración de la Base de Datos de Construcciones Verbales Fijas}

La base de datos consta actualmente de 29.000 CVF que se han ido recopilando en dos fases bien diferenciadas:

\subsection{Primera fase: recopilación en diccionarios españoles}

En esta primera fase (que todavía sigue en proceso abierto de recopilación), se ha llevado a cabo, una recopilación de las expresiones usadas en España a partir de diccionarios españoles. Conviene destacar que actualmente, de

2 Diccionario de Uso del Español. Cada uno de los ejemplos de tipos de UF que presentamos a continuación ha sido encontrado en este diccionario. De cara a aportar mayor fiabilidad a nuestro estudio, en este artículo indicaremos para cada expresión las siglas del diccionario, entre paréntesis, en el que se haya recopilado esa expresión. 
las 29000 CVF de la BD 20.600 pertenecen a expresiones recopiladas en diccionarios españoles y no marcadas como variantes diatópicas hispanoamericanas. ${ }^{3}$ De éstas 20.600 expresiones:

- 18.000 figuran en diccionarios monolingües y bilingües: Diccionario de la Real Academia Española de la lengua, (RAE); Diccionario de uso del español, (DUE); Diccionario temático de locuciones francesas con su correspondencia española, (DT); Diccionario fraseológico del español actual, (DFDEA); Diccionario fraseológico del español moderno, (DFDEM); Diccionario del español actual, (DEA); Diccionario temático de frases hechas, (DTDFH); Enciclopedia Planeta Multimedia, edición 2010 DVD-ROM, (EPM); Gran diccionario Larousse Español-Francés / Francés-español, (LBI); Gran Diccionario Espasa EspañolFrancés / Francés-español, (DEBI); Jergas, Argot y Modismos (LIBSA); etc.

- 2.600 son expresiones usuales o conocidas pero que no han sido recopiladas en los diccionarios que hemos analizado. ${ }^{4}$ Para su recopilación e inclusión en la BD hemos contado inicialmente con la competencia fraseológica del grupo de investigadores que forman FRASYTRAM. Posteriormente hemos verificado la presencia de estas expresiones en internet. Para ello, hemos realizado la búsqueda de estas expresiones en su forma canónica no lematizada, es decir en infinitivo únicamente, en el servidor google: https://www.google.es/. Esta búsqueda nos permite indicar para cada expresión el número de ocurrencias que se han encontrado en internet. Ocurrencias que han sido indicadas entre paréntesis después del signo $\emptyset$. Como botón de muestra, reproducimos a continuación algunas de estas expresiones españolas usuales por lo común pero que no han sido por ahora, repetimos, incluidas en los fondos de los diccionarios españoles analizados. [abrirse, cortarse] (alguien) las venas (suicidarse, $\varnothing,{ }^{5}$ 184.000); tirarse (alguien) a la piscina (actuar con decisión, resueltamente, $\emptyset$, 500.000); estar (alguien/algo) en medio como el jueves (estorbar, Ø, 25.000); salir (alguien) del armario (declarar su homosexualidad, $\emptyset$, 450.000); faltar (a alguien) un hervor (, 15.000$)$; ganarse (alguien) el cocido (trabajar para asegurarse el sustento, $\varnothing, 19.000)$; etc.

3 Evidentemente esto no significa que estas expresiones no sean conocidas o usadas en Hispanoamérica, de hecho estamos ya trabajando en un proyecto de cara a poder establecer el uso real de un núcleo común usual de expresiones españolas en España y en diferentes países de Hispanoamérica.

4 Para indicar que estas CVF no figuran en los diccionarios analizados, hemos incluido después de la definición el símbolo $\emptyset$.

5 El signo $\emptyset$ indica que la expresión no aparece en los diccionarios consultados para hacer la BD. 


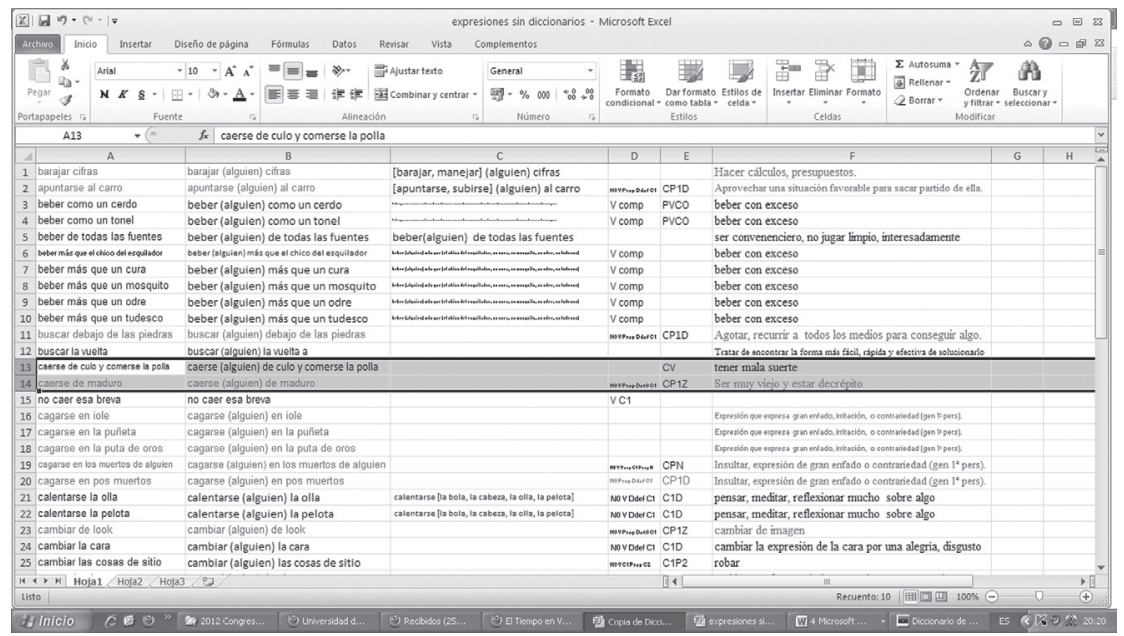

Figure 1: Captura de Pantalla $n^{\circ}$

\subsubsection{Presencia de CVF diatópicas regionales en los diccionarios españoles}

Durante la elaboración de la BD realizada inicialmente a partir de diccionarios españoles, encontramos también en estas obras una pequeña serie de expresiones marcadas en los diccionarios como pertenecientes a un uso regional español. En todas las lenguas existen también en el espacio geográfico que delimita su uso, variantes fraseológicas diatópicas que aparecen irremediablemente en cada una de las regiones y con las que los usuarios de esa(s) lengua(s) expresan los mismos conceptos en los actos de comunicación con unas combinaciones plurilexicales que utilizan elementos léxicos o culturales propios a esa determinada región sumándole de esa forma un matiz local que no conocen o no utilizan las demás áreas geográficas. Así encontramos un número reducido de expresiones regionales: ${ }^{6}$

- en los diccionarios españoles más referenciales (como el DRAE o el DUE) calificadas como de uso regional en España: echar (alguien) los kiries (vomitar, RAE, And); estar (alguien) a ruche (estar sin dinero; DUE; Extr., Gran., Mur., Rioj., Vall.); sentar (alguien) las botas (colocar los toneles a lo largo de las paredes de la bodega, DUE, Jerez); rascarse (alguien) el bolso (gastarse el dinero de mala gana, DFDEA, regional), etc.

6 El número no pasa por ahora de 30 expresiones en el primer grupo y de 150 en el caso del segundo grupo de diccionarios regionales. 
- en otros diccionarios españoles, de corte más regional utilizados para ampliar la $\mathrm{BD}$, algunas expresiones también con una procedencia y un uso más regional que generalmente no son conocidas en las otras regiones españolas. Ej: el Nuevo Diccionario Etimológico Aragonés (NDEA) hacer (alguien) bolsa (ahorrar, economizar, guardar dinero); ser (alguien) del brazo arremangado (ser valiente, esforzado, intrépido); ser (alguien) del mismo brazo (tener la misma posición económica sobre todo en hacienda); estar (alguien) como los burros en el mes de marzo (retozón, juguetón); etc.

el Diccionario de Expresiones y Refranes del español de Canarias (DEREC) ir (alguien) a darle agua a la becerra (LP, ir a ver a la novia); jalar (alguien) la brasa pa(ra) su sardina (aprovechar una persona para lo que le interesa o importa, la ocasión o coyuntura que se le ofrece).

\subsubsection{Presencia de CVF diatópicas hispanoamericanas en los diccionarios españoles}

Durante esa misma fase, se han extraído también en los diccionarios españoles analizados, un número ya bastante apreciable de expresiones marcadas en los diccionarios como pertenecientes a un uso diatópico, más concretamente al español de Hispanoamérica. Si en todas las lenguas aparecen variantes regionales, en el caso de lenguas como el español, el francés, el inglés, etc. que son hablados en numerosos países por decenas y/o centenas de millones de personas, existen a su vez numerosísimas creaciones idiosincrásicas propias a cada país, cada cultura y a sus referentes culturales. Se trata de las expresiones o de las variantes utilizadas en los diferentes países en los que estas lenguas son lenguas oficiales y son habladas por gran parte de su población. Estas UF son creaciones propias a cada uno de estos países cuyo(s) significado(s) a menudo a pesar de tener componentes léxicos del español resultan opacos para los usuarios españoles debido en gran medida a que no pertenecen a la competencia fraseológica de los usuarios españoles.

En este caso, por ahora se han recopilado $1100 \mathrm{CVF}$, en los diccionarios españoles consultados, acompañadas de anotaciones de uso diatópico del español de Hispanoamérica. Por ej:

- agarrar alguien viaje (Aceptar una propuesta; RAE, Arg, Par, Perú y Ur); agarrarse alguien del chongo (reñir, pelear, RAE, Méx ${ }^{7}$ ); apagar alguien la luz (irse alguien el último de un lugar o reunión, DTDFH; Argentina); azotar alguien la

7 En este trabajo, cuando reproduzcamos las anotaciones presentes en los diccionarios, respetaremos fielmente éstas mismas incluso cuando varios diccionarios utilicen siglas diferentes para referirse a un mismo país. Así el DRAE para hablar de México utiliza (Méx) y el DUE (Méj). 
lana (dar dinero, pagar, RAE, El Salv); apuntarse alguien un poroto (anotarse o apuntarse un tanto en el juego, o un acierto en cualquier actividad, RAE, Am Me); caer a alguien el chahuistle (tener muy mala suerte, DUE, Méj); caerse alguien de la cama (Ser muy ingenuo, RAE; Cuba); descubrir alguien el agua $\left[\right.$ fría, tíbia] ${ }^{8}$ (presentar como novedoso algo ya muy conocido, RAE, Cuba); doblar alguien la esquina (cambiar de tema, pasar a tratar un asunto o tema diferente, DTDFH, Cuba); ganarse alguien los porotos (ganarse la vida, RAE, Chile); gastar alguien pólvora en gallinazo (hacer esfuerzos por algo o alguien que, en realidad, tiene poca importancia, RAE, Ec); pedir alguien cacao (pedir clemencia o benevolencia, DUE, Col, Guat Méj); etc.

Estos pocos ejemplos nos permiten observar que los diccionarios españoles analizados incorporan CVF de todos los países hispanoamericanos. Por orden cronológico (de los ejemplos de CVF diatópicas anteriores), observamos en los ejemplos CVF de Argentina, Paraguay, Perú, Uruguay, México, El Salvador, república Dominicana, Cuba, Chile, Ecuador, Venezuela, Nicaragua, Guatemala, etc. Esta diversidad geo lingüística se puede apreciar también en la captura de pantalla de la Base de Datos que estamos elaborando y en la que hemos

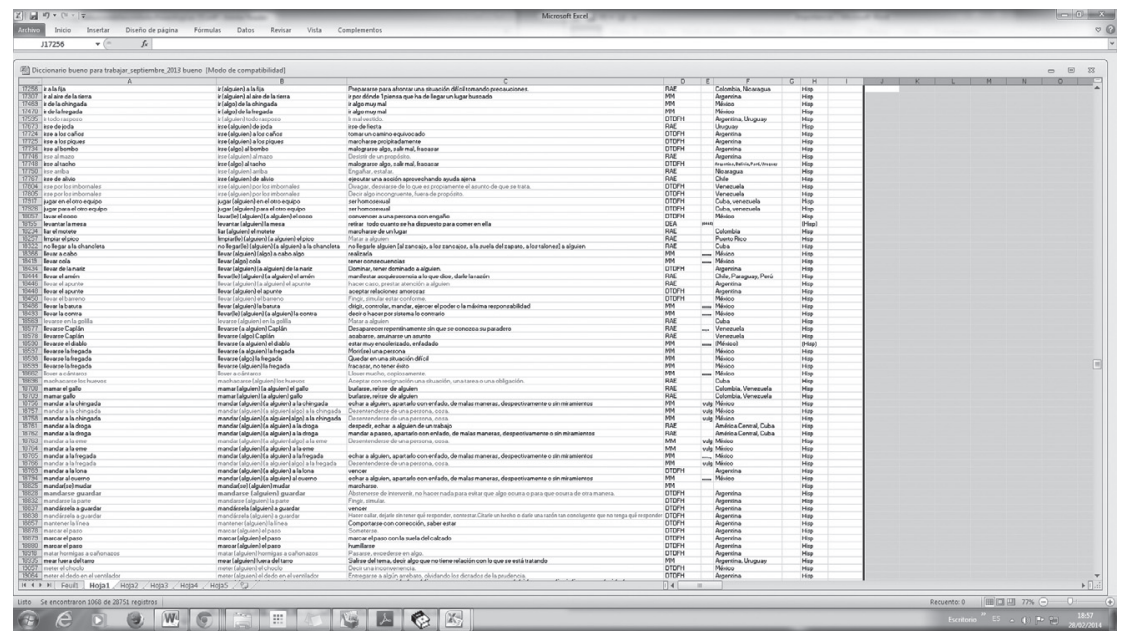

Figure 2: Captura de pantalla $n^{\circ} 1$

8 Cada vez que una CVF presente variantes sintácticas es decir pueda tener variantes léxicas, escribiremos entre corchetes estas posibles variantes. En el caso de la CVF descubrir (alguien) el agua [fría, tibia] los corchetes indican que existe la CVF descubrir (alguien) el agua fría y la CVF descubrir (alguien) el agua tibia. 
seleccionado a través de los filtros del programa las CVF de la BD recopiladas en diccionarios españoles y que tienen un origen y un uso hispanoamericano. ${ }^{9}$

Sin embargo, retomando las cifras que hemos indicado al principio, esto significa que los diccionarios españoles analizados incorporan unas 1100 CVF diatópicas de Hispanoamérica de un total de 20.600 CVF. Es decir que solamente un $0.5 \%$ de las CVF presentes en los fondos de los diccionarios españoles son expresiones pertenecientes al español de américa usadas y creadas en Hispanoamérica.

Por países, el origen de las CVF hispanoamericanas que figuran en los diccionarios españoles analizados es el siguiente: Argentina: 367; agarrar (alguien) viaje (aceptar una propuesta; RAE, Arg, Par, Perú y Ur); Bolivia: 22; dar alguien capote $a$ alguien (dar a alguien muchas palmadas en son de castigo; RAE, Bol); Chile 99; andar alguien de maletas (estar de mal humor; RAE; Chile); Colombia: 26; dar (alguien) en el bocín (acertar, dar en el blanco, DUE); Costa Rica: 44; buscar alguien la comba al palo (esforzarse por resolver una situación difícil; RAE; Costa rica); Cuba: 118; doblar alguien la esquina (cambiar de tema, pasar a tratar un asunto o tema diferente, DTDFH, Cuba): estar alguien más pelado que un plátano (estar sin dinero, DTDFH); Ecuador: 36; darse alguien teja (presumir, jactarse, hacer alarde de, RAE); El Salvador: 33; no dar alguien patada sin mordida (ser convenenciero, interesado, RAE); Guatemala: 40; amarrar alguien zope (Vomitar, RAE); Honduras: 33; echar alguien el ala (enamorar a una mujer; RAE); México: 229; caer (a alguien) el chahuiscle (sobrevenirle a alguien un mal, molestia, RAE, Méx); Nicaragua: 29; irse alguien arriba (engañar, estafar, RAE); Panamá: 9; Paraguay: 7; (Conversar alguien de bueyes perdidos y vacas encontradas (conversar sobre un tema cualquiera independientemente de su trascendencia, DTFFH); Perú: 61; meter (alguien) yuca (engañar, burlar a alguien, DTDFH); Puerto Rico: 27; República Dominicana: 20 caer burros aparejados (llover copiosamente, LIBSA); tener alguien tabaco en la vejiga (ser muy valiente, DRLE); Uruguay: 68; estar alguien del tomate (estar loco, DTDFH); Venezuela: 65; gastar alguien pólvora en zamuros (hacer esfuerzos por algo o alguien que, en realidad, tiene poca importancia, RAE).

En el fondo, esta escasa presencia en los diccionarios de las expresiones diatópicas españolas no responde sino a las pautas seguidas por la investigación fraseológica española que si bien ha investigado considerablemente las Unidades Fraseológicas lo ha hecho centrándose sobre todo en las UF del español peninsular. Estas expresiones diatópicas españolas están siendo por fin recopiladas

9 Sería interesante poder analizar la inclusión de las CVF elegidas por los diccionarios para poder averiguar el motivo de inclusión de estas formas y no de otras. Por su frecuencia de uso, por su registro de lengua, etc. 
con más exhaustividad en su ámbito territorial de influencia, pues desde hace unos años se están elaborando diccionarios en los países hispanoamericanos que recopilan los términos y las numerosas expresiones características de cada uno de esos países. No se han realizado sin embargo ni recopilaciones exhaustivas (que reúnan sino todas, al menos una gran parte de estas UF diatópicas de cara a poder conocerlas, ni análisis contrastivos que permitan observar las similitudes y las diferencias sintácticas y léxicas entre estas creaciones diatópicas con todos sus referentes culturales idiosincrásicos y conocer sus usos regionales o geográficos. La mayoría de los trabajos de investigación que se han realizado y se siguen llevando a cabo, en el ámbito de la fraseología se han llevado a cabo, con expresiones que pertenecen al país del investigador o que contrastan algún pequeño grupo de expresiones.

\subsection{Segunda fase: recopilación en diccionarios hispanoamericanos}

Durante el desarrollo de la primera fase, la investigación llevada a cabo para realizar el proyecto de Investigación FFI2011-24310 «Estudio lingüístico, diatópico y traductológico de las construcciones verbales fijas más usuales en español», proyecto en el que entre otras investigaciones buscamos CVF mexicanas y argentinas, nos hizo buscar diccionarios hispanoamericanos y nos permitió detectar por un lado la reciente aparición de numerosos diccionarios elaborados en Hispanoamérica, así como la magnitud del fenómeno fraseológico diatópico en el continente Americano. Esta segunda fase, que está realizándose actualmente y en la que estamos sobre todo recopilando expresiones diatópicas en diccionarios hispanoamericanos, ${ }^{10}$ ha permitido incorporar a la BD otras $8000 \mathrm{CVF}$, es decir que la BD ya contiene más de 9000 CVF del español de Hispanoamérica. Ej: apelar (alguien) a las de gaviota (huir precipitadamente de un sitio, Argentina, DFHA); adobar (alguien) la piñata (preparar la comida, Argentina, DFHA); aflojar (alguien) mecate (disminuir alguna restricción, ser más permisivo, DdAm, Honduras, México, Venezuela); hablar (alguien) por el ruido de las nueces (hablar sin

\footnotetext{
10 Referencia de los diccionarios Hispanoamericanos utilizados.

AoMex $=$ Diccionario breve de mexicanismos.

DHDA = Diccionario del habla de los argentinos.

DDA = Diccionario del español de Argentina .

DdAm = Diccionario de americanismos.

DEUEM = Diccionario del español usual en México

DFDHA = Diccionario fraseológico del habla argentina.
} 
saber por lo que los otros dicen, DTDFH, Chile); hacer (alguien) lunes de zapatero (prolongar el fin de semana, DRLE, Colombia); pedir (alguien) cacao (pedir clemencia o benevolencia, DUE, Col, Guat Méj); etc.

El origen de estas expresiones es a su vez el siguiente: Argentina: 2440; abrirse (alguien) de gambas (1. referido a un problema o dilema, desentenderse o excusarse, DFHA; 2. eludir responsabilidades, DFHA; 3. referido a una mujer, tener relaciones sexuales, GDLA, grosero; 4. aguantar, sufrir resignadamente, soportar con resignación una injusticia, un abuso, pena, castigo, GDLA, coloquial); Bolivia: 237; agarrar (alguien) la copa (dedicarse a consumir bebidas alcohólicas, DdAm); Chile: 658; arrastrar (una chiquilla) la bolsa del pan (no haber alcanzado una mujer la edad para mantener relaciones sexuales, DdAm); Colombia: 375; caer la porroca a alguien (tener mala suerte, DdAm); Costa Rica: 241; agarrar (alguien) (a alguien) de chancho (burlarse de alguien, DdAm); Cuba: 826; estar (alguien) más pelado que un plátano (estar sin dinero, DTDFH); Ecuador: 192; cargarse (alguien) el año (reprobar un estudiante de primaria o secundaria el curso lectivo, DdAm); El Salvador: 43; no dar (alguien) patada sin mordida (ser convenenciero, interesado, RAE); Guatemala: 381; apagar (a alguien) el ocote (perder el entusiasmo por algo, DdAm); Honduras: 535; agarrar (alguien) palco (tomarse demasiada confianza, DdAm); México:1997; abrir (alguien) los ojos (perder la inocencia, DEUEM); Nicaragua: 481, cantar (alguien) el volado (descubrir un secreto, DdAm); Panamá: 231; dar (a alguien) lo mismo Chana que Juana (dar igual una cosa que otra, DdAm); Paraguay: 57; conversar (alguien) de bueyes perdidos y vacas encontradas (conversar sobre un tema cualquiera independientemente de su trascendencia, DTFFH); Perú: 330; meter (alguien) yuca (engañar, burlar a alguien, DTDFH); Puerto Rico: 629; aguantar (alguien) el caballito (controlar una persona, frenarla, DdAm); República Dominicana: 425; tener (alguien) tabaco en la vejiga (ser muy valiente, DRLE); Uruguay: 236; estar (alguien) del tomate (estar loco, DTDFH); Venezuela: 481; alzarse (alguien) con el coroto, (usurpar alguien el poder, DdAm).

Vemos que por ahora en los dos casos, el mayor número de expresiones hispanoamericanas recopiladas corresponde numéricamente a Argentina, México y Cuba. Ese resultado coincide tanto en el caso de los diccionarios españoles, (en los que hemos catalogado: Argentina (367), México (229) y Cuba (117)), como en el de los diccionarios hispanoamericanos, en los que se han catalogado (Argentina (2240), México (1997) y Cuba (826). Las cifras de las CVF hispanoamericanas presentes en los diccionarios españoles variarán muy poco pues la fase de recopilación en estos diccionarios está casi terminada. Tal no es el caso de los diccionarios hispanoamericanos, por lo que el número de CVF diatópicas sufrirá con toda seguridad un cambio apreciable en los datos generales de la BD. 
Table 1: \#\#\# add caption \#\#\#

\begin{tabular}{|c|c|c|c|c|}
\hline Año y publicación & Total BD & $\begin{array}{l}\text { DUE + } \\
\text { DRAE }\end{array}$ & $\%$ & $\begin{array}{l}\text { Expresiones } \\
\text { Hispanoamericanas }\end{array}$ \\
\hline $\begin{array}{l}2006 \text { (Colocaciones y fraseología } \\
\text { en los diccionarios, publicado en } \\
2008 \text { ) }\end{array}$ & 13.350 & 8.253 & $60 \%$ & - \\
\hline $\begin{array}{l}2009 \text { (Passeurs de mots, } \\
\text { publicado en 2011) }\end{array}$ & 15.913 & 9.090 & $57 \%$ & - \\
\hline 2010 (Enfoques de teoría) & 18.000 & 9.960 & $56 \%$ & - \\
\hline 2013 México (comunicación oral) & 27.200 & 11.900 & $44 \%$ & $8.000=27 \%$ \\
\hline 2014 (datos actuales) & 29.000 & 12.741 & $43 \%$ & $9.000=31 \%$ \\
\hline
\end{tabular}

Ya hemos comentado en artículos anteriores el escaso tratamiento que los diccionarios españoles daban a las CVF del español peninsular (Mogorrón 2006, 2009, 2010, 2013) y como botón de muestra exponíamos el número de CVF de la $\mathrm{BD}$ que aparecían en los dos diccionarios más usuales de la lengua española (El Diccionario de la Real Academia española de la lengua (RAE) y el Diccionario del Uso del español (DUE) que reproducimos a continuación:

Se puede apreciar claramente que el porcentaje de CVF de la Base de datos contenidos en los dos diccionarios más usuales:

- disminuye claramente a medida que se van vaciando otros diccionarios españoles como el DFDEA, el DFDEM que incluyen en sus fondos expresiones posiblemente más actuales o que pueden pertenecer a registros considerados más populares, familiares, coloquiales.

- baja considerablemente y pasa a ser netamente inferior al 50\% a partir de la inclusión de las CV diatópicas. Pensamos a la vista de la evolución de los datos de la Base de datos de CVF, que el porcentaje seguirá a todas luces disminuyendo progresivamente.

\section{Tratamiento de las variantes diatópicas en los diccionarios españoles}

Deseamos a continuación mostrar, (una vez que ha quedado claro, con las cifras globales ofrecidas), el escaso tratamiento que dan los diccionarios españoles e Hispanoamericanos a las CVF del español peninsular y a las CVF diatópicas pertenecientes al español hablado en los países hispanoamericanos. Para ello, analizaremos la presencia en una docena de diccionarios españoles 
e hispanoamericanos ${ }^{11}$ de las CVF parasinónimas de llover mucho, con fuerza, intensamente recopiladas hasta la fecha en la Base de datos y de aquellas que hemos conseguido encontrar con el mismo significado en internet. Se trata de un fenómeno atmosférico frecuente y recurrente en España y en todos los países hispanoamericanos por lo que es de suponer que ese concepto tendrá muchas formas de describirse expresivamente con expresiones fijas. ${ }^{12}$ De hecho ya existen en español peninsular varias expresiones para describirlo, ¿pero se usan en Hispanoamérica y aparecen en los diccionarios hispanoamericanos? Finalmente ¿qué expresiones se usan en los países hispanoamericanos?

Hemos clasificado las CVF parasinónimas de llover mucho, intensamente en los cuatro grupos siguientes:

- CVF del español peninsular que aparecen en diccionarios españoles:

- [caer, llover] capuchinos de bronce (DDFEJC); [caer, llover] chuzos (de punta) (DUE); [descargar, desgajarse] [el cielo, las nubes] (DUE); llover a mares (DUE); llover a cántaros (DUE); llover a jarros (DUE); llover a mares (DUE); llover más que [cuando, el día que] enterraron [a Zafra, al Zafra] (EPM).

- CVF del español peninsular recopiladas en internet:

- en Navarra y Guipuzcoa, está jareando $;^{13}$ en Santiago de Compostela, llover a barriles; en Canarias llover más que cuando se casó Abraham ${ }^{14}\left(\right.$ DEREC $\left.^{15}\right)$; llover más que el año que el barranco se llevó a la puerca (DEREC), etc.

- CVF del español de hispanoamérica recopiladas en diccionarios hispanoamericanos.

11 Los diccionarios usados para el análisis son a nuestro entender de los más representativos de su tipo. Se trata de DUE; DRAE; EPM; DTDFH; LBI; DDAMERC; AOMEX; DDEUM; DDMEX; GDHA; DDA::

12 La prueba de que esta situación meteorológica es más que frecuente en todos los países es por ejemplo que en francés tenemos por ejemplo la expresión pleuvoir comme vache qui pisse (GR) pleuvoir à verses (GR); pleuvoir à seaux (GR); etc y que en diferentes países francófonos encontramos: en francés de Quebéc pleuvoir à boire debout (BFQS); tomber comme des clous (BFQS); en Suiza pleuvoir des seilles (BFQS/S); en Côte d'Ivoire tomber une pluie de mangues (SL) o tomber une pluie des vaches (SL); etc. Recordemos que una de las funciones de las expresiones fijas es a menudo la de realzar la expresividad de lo que se quiere transmitir y que el locutor renunciando a usar un término no marcado o neutro desea incrementar con una expresión la fuerza expresiva y comunicativa acerca de un concepto o de una noción con esa fórmula.

13 Hemos encontrado más de 40.000 referencias de está jarreando en Internet

14 Existen variantes con el nombre del personaje que se casó. En Valladolid llover más que cuando se casó Morrondo, en Castilla la Mancha , llover más que cuando se casó Neo, etc.

15 Diccionario de expresiones y refranes del español de Canarias. 
- [caer, llover] burros aparejados (DDAMER, República Dominicana); caer sapos de punta (DFHA, Argentina); caer sapos panza arriba (DFHA; Argentina); caer soretes de punta (DFHA; Argentina); llover a baldazos (GDHA; Norte de Argentina ${ }^{16}$ ); llover a baldes (GDHA; Norte de Argentina); estar cayendo [bigornias, pingüinos] de punta (GDHA; Argentina).

- CVF del español de Hispanoamérica recopiladas en internet.

- Abrirse el cielo ${ }^{17}$ (Puerto Rico); caer raíles de punta (internet, Cuba); estar cayendo el cielo (internet, Venezuela); estar cayendo un palo/coñazo de agua (internet, Venezuela); estar lloviendo pescados y hasta ballenas (Internet, Guatemala); llover de abajo para arriba ${ }^{18}$ (Argentina, Costa Rica); llover sapos y culebras (internet, El Salvador).

A continuación hemos trasladado los resultados de la búsqueda realizada para averiguar la presencia de las CVF en los diccionarios ya mencionados en la nota a pie de página $\mathrm{n}^{\circ} 12$ en el siguiente cuadro. Para facilitar la lectura y la interpretación de los resultados, las CVF están ordenadas siguiendo la clasificación anterior en cuatro bloques.

Table 2: \#\#\# please add caption \#\#\#

\begin{tabular}{|c|c|c|c|c|c|c|c|c|c|c|c|c|}
\hline Expresiones & DUE & D & $\mathbf{E}$ & D & D & $\mathbf{L}$ & D & A & DEUEM & DD & G & D \\
\hline usuales españolas & & $\mathbf{R}$ & $\mathbf{P}$ & $\mathbf{T}$ & $\mathbf{F}$ & B & D & 0 & & $\mathbf{M}$ & D & D \\
\hline recopiladas en & & A & M & D & D & I & A & $M$ & & $\mathbf{E}$ & $\mathbf{H}$ & A \\
\hline \multirow[t]{2}{*}{ diccionarios } & & $\mathbf{E}$ & & $\mathbf{F}$ & $\mathbf{E}$ & & ME & $\mathbf{E}$ & & $\mathbf{x}$ & A & \\
\hline & & & & H & A & & $\mathbf{R}$ & $\mathrm{x}$ & & & & \\
\hline $\begin{array}{l}\text { [caer, llover] } \\
\text { capuchinos (de } \\
\text { bronce) }\end{array}$ & - & + & - & + & - & - & - & - & - & - & - & - \\
\hline $\begin{array}{l}\text { [caer, llover] chuzos } \\
\text { (de punta) }\end{array}$ & + & + & + & + & + & + & - & - & - & - & - & - \\
\hline $\begin{array}{l}\text { [descargar, } \\
\text { desgajarse] [el } \\
\text { cielo, las nubes] }{ }^{1}\end{array}$ & + & + & - & + & - & + & - & - & - & - & - & - \\
\hline $\begin{array}{l}\text { descargar el } \\
\text { nublado }^{1}\end{array}$ & + & + & - & + & - & - & - & - & - & - & - & - \\
\hline llover a cántaros & + & + & + & + & + & + & - & - & + & - & + & - \\
\hline llover a jarros & + & + & - & + & + & - & - & - & - & - & - & - \\
\hline
\end{tabular}

(Continued)

16 Gran diccionario de habla Argentina.

17 www.wordreference.com/es/translation.asp

18 www.wordreference.com/es 
Table 2: (Continued)

\begin{tabular}{|c|c|c|c|c|c|c|c|c|c|c|c|c|}
\hline llover a mares & + & + & + & + & + & $a d v$ & - & - & - & - & - & - \\
\hline llover a más y mejor & + & + & - & + & $\mathrm{adv}$ & $\mathrm{adv}^{1}$ & - & - & - & - & - & - \\
\hline $\begin{array}{l}\text { llover más que } \\
\text { [cuando, el día que] } \\
\text { enterraron [a Zafra, } \\
\text { al Zafra] }\end{array}$ & - & - & + & + & - & - & - & - & - & - & - & - \\
\hline $\begin{array}{l}\text { Expresiones diatópic } \\
\text { del español peninsul } \\
\text { recopiladas }\end{array}$ & & & & & & & & & & & & \\
\hline $\begin{array}{l}\text { estar jareando } \\
\text { (Navarra y } \\
\text { Guipuzcoa) }\end{array}$ & - & - & - & - & - & - & - & - & - & - & - & - \\
\hline $\begin{array}{l}\text { llover a barriles } \\
\text { (Santiago de } \\
\text { Compostela) }\end{array}$ & - & - & - & - & - & - & - & - & - & - & - & - \\
\hline $\begin{array}{l}\text { llover más que } \\
\text { cuando se casó } \\
\text { Abraham (DEREC; } \\
\text { canarias) }\end{array}$ & - & - & Zafra & - & - & - & - & - & - & - & - & - \\
\hline $\begin{array}{l}\text { llover más que el } \\
\text { año que el barranco } \\
\text { se llevó a la puerca } \\
\text { (DEREC, Canarias) }\end{array}$ & - & - & - & - & - & - & - & - & - & - & - & - \\
\hline
\end{tabular}

\section{Expresiones}

hispanoamericanas

recopiladas en

\section{diccionarios}

[Caer, llover] burros aparejados

(R Dominicana)

Caer sapos de punta (Argentina)

Caer sapos panza

arriba (argentina)

[caer, Llover]

soretes de punta

(Argentina)

estar cayendo

[bigornias,

pingüinos] de

punta (Argentina)

llover a [baldes,

baldazos]

(Argentina) 
Table 2: (Continued)

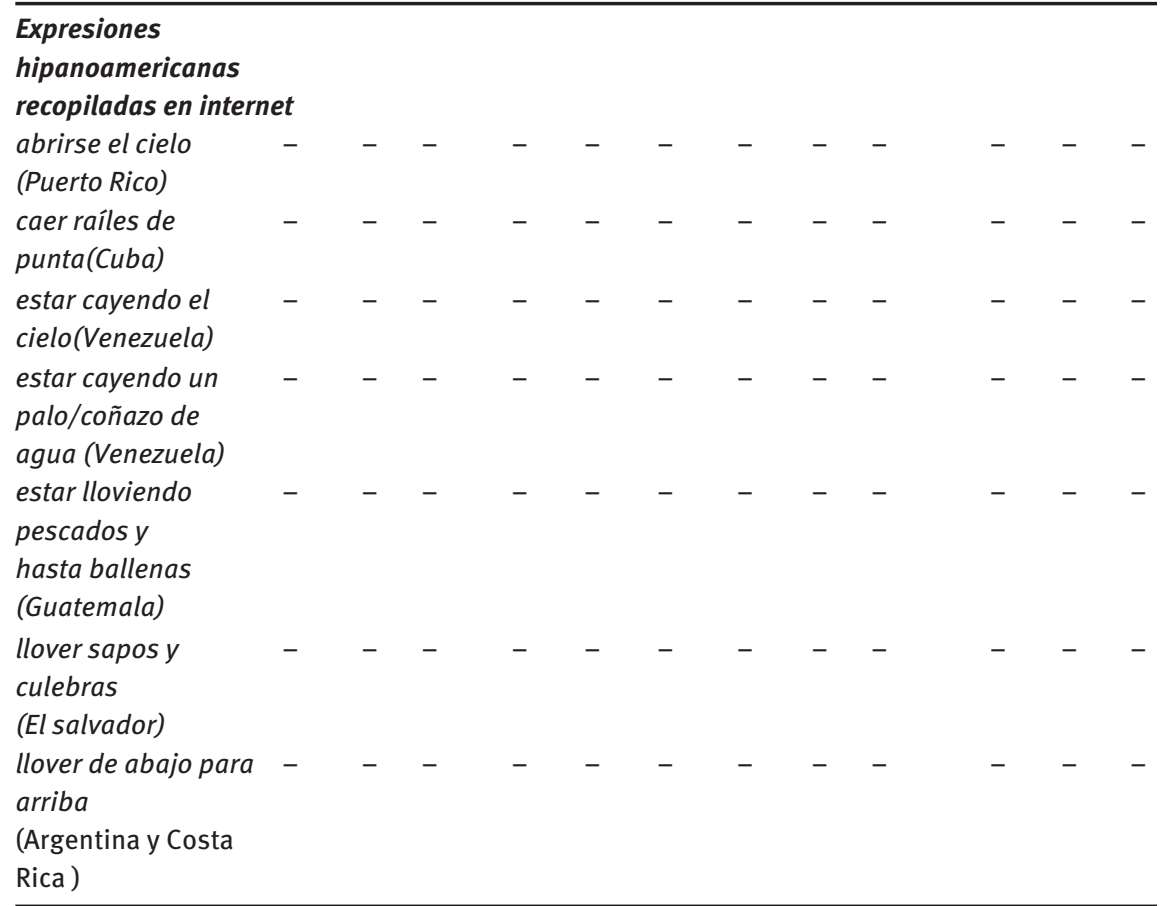

Globalmente la primera observación que se impone es que los diccionarios no recogen muchas de las CVF recopiladas en la fase de búsqueda de esta investigación, bien en la BD bien en internet. Es verdad que generalmente, los diccionarios tardan en incorporar expresiones, términos o nuevas acepciones es decir que suelen incluir usos consagrados por el uso de numerosas generaciones. Existe un desfase considerable entre el continuo e imparable proceso de remodelación de los actos de comunicación constantemente obligados a readaptarse y el material que los lexicógrafos insertan en los diccionarios. En efecto, por un lado están las UF de la lengua clásica, a menudo ya en desuso por pertenecer a un uso no ya de otras generaciones sino también a una concepción diacrónica y que por lo tanto ya no son representativas de los usos y estados lingüísticos que se van sucediendo. Por otro lado, se halla el uso presente, marcadamente innovador de la lengua, que se debe ir transformando y adaptando a los constantes cambios que se producen en la lengua por los usos generacionales y las diferentes necesidades y realidades sociales que se plasma en nuevos vocablos y expresiones.

Pero además en el caso de lenguas como el español, el francés, el inglés, el portugués utilizadas como lenguas oficiales en numerosos países, la realidad social y las diferencias geográficas producen numerosos términos o expresiones 
características de cada país. Vemos que esas expresiones no figuran en los diccionarios considerados más referenciales como el DRAE o el DUE con un carácter más unificador, clásico y conservador. También observamos que los diccionarios pertenecientes a cada país hispanoamericano se limitan generalmente a recopilar algunas expresiones y a señalarlas como pertenecientes a esa variedad del español hispanoamericano sin informar si esas expresiones son usuales en otros países de Hispanoamérica.

Así por ejemplo el DDMexicanismos señala que la CVF comer gente (Censurar o hablar mal de las personas) se usa en México y el DdAm indica que se usa en Nicaragua y en Costa Rica. Lo mismo sucede con la CVF ganarse los fríjoles (Ganarse la vida) señalada también por el DDmexicanismos como mexicanismo mientras que el DdAm señala que su zona de influencia es Nicaragua, Cuba y Ecuador. ${ }^{19}$

Se puede apreciar que:

- Las CVF del español peninsular presentes en los diccionarios españoles no aparecen en los diccionarios hispanoamericanos excepción hecha de llover a cántaros que aparece también en el Diccionario de americanismos y en el Diccionario del español usual en México.

- Las expresiones del español peninsular encontradas en internet y en diccionarios menos referenciales no aparecen en ninguno de los 12 diccionarios del español peninsular o hispanoamericanos utilizados como herramienta de trabajo.

- Las expresiones diatópicas hispanoamericanas presentes en los diccionarios hispanoamericanos no aparecen en los diccionarios españoles con la excepción de llover a baldes que aparece en el DTDFH (diccionario que casualmente contienen un porcentaje más elevado de expresiones diatópicas hispanoamericanas).

- Las expresiones diatópicas hispanoamericanas encontradas en internet y en diccionarios menos referenciales no aparecen en ninguno de los diccionarios del español peninsular o hispanoamericano analizados.

Así pues, los diccionarios hispanoamericanos no incluyen a menudo expresiones del español estándar o común e incluyen generalmente expresiones que pertenecen al país en el que se elabora el diccionario. Pero significa eso que no se usan en hispanoamérica las expresiones usuales españolas?

19 Hasta la fecha era harto difícil poder encontrar ese tipo de información pero en la actualidad algunas obras como el Diccionario de Americanismos intentan suplir esa carencia de información de uso multinacional de algunas expresiones. 
Volviendo a las expresiones parasinónimas de llover mucho, intensamente, hemos consultado hablantes de Argentina, Colombia y Perú todos ellos profesores universitarios o estudiantes en fase doctoral que nos han permitido averiguar que:

- en Argentina se conoce y usa, (a parte de las CVF que ya hemos señalado como de uso argentino; las siguientes expresiones: llover a cántaros y llover a mares pertenecientes al primer grupo, caer sapos de punta del tercer grupo y estar cayendo el cielo, llover sapos y culebras del último grupo.

- en Colombia y en Perú se conoce y se usa llover a cántaros y llover a mares;

Pensamos por lo tanto, que si son usuales en esos países, los diccionarios hispanoamericanos deberían al menos incorporarlos en sus fondos para reflejar el uso real de la lengua española en esos países.

\section{Fenómenos lingüísticos que se podrán tratar con este tipo de Base de datos}

La BD asimismo también permitirá tratar varios fenómenos lingüísticos que aparecen en las UF tales como la variación paradigmática, la parasinonimia, la polisemia. Todos estos fenómenos afectan a numerosas UF y son tan amplios que plantean numerosos problemas y quebraderos de cabeza a los lingüistas, aprendices de lenguas y traductores.

En Mogorrón 2010 hemos demostrado como más del 50\% de las CVF de una BD de 18.000 unidades del español peninsular presentaban algún tipo de variación generalmente en los sustantivos o los verbos.

A partir de la BD elaborada con la recopilación de CVF de los diccionarios, estamos convencidos de que herramientas modernas con el potencial de internet permitirían ampliar el número de CVF con variación y polisemia. Para la variación de las UF diatópicas, por ahora tenemos anotadas unos 1.600 casos de variación diatópica en la BD. Tendremos que terminar de vaciar los diccionarios hispanoamericanos para estar en medida de presentar cifras más reales. Asimismo, convendrá pensar si aplicamos la misma restricción geográfica para la variación paradigmática-diatópica tal y como hicimos con las expresiones españolas. Es decir si solamente tendremos en cuenta la variación que se produzca en un único país o si por el contrario ampliáremos el abanico de posibles variaciones a posibles variantes en diferentes países hispanoamericanos aumentando entonces también el número de variantes. 


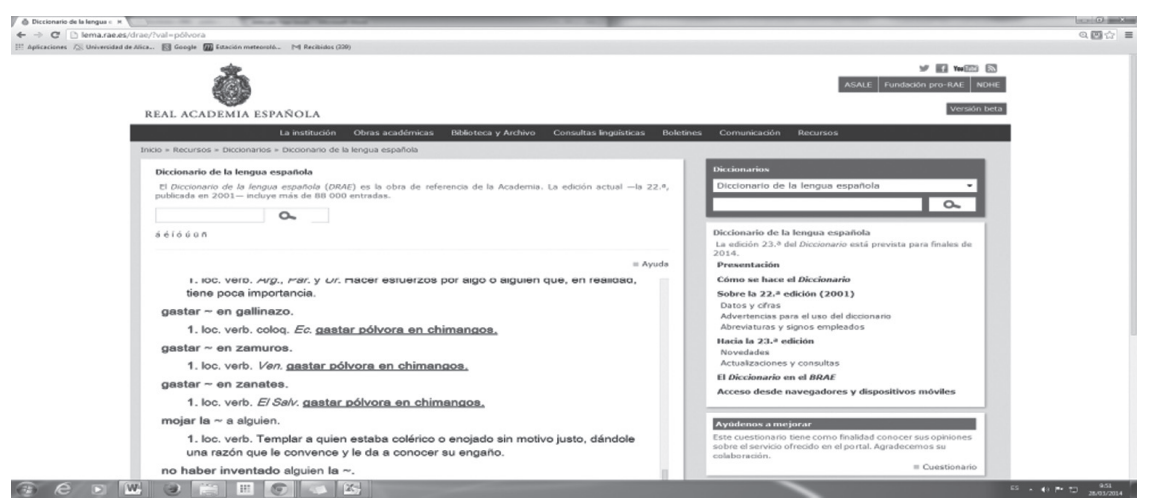

Figure 3: Captura de pantalla ${ }^{\circ}$

En el primer grupo tendríamos variaciones del tipo abrirse (alguien) de [gambas, piernas] (GDLA; Argentina) (1. referido a una mujer, tener relaciones sexuales; 2. aguantar, sufrir resignadamente, soportar con resignación una injusticia, un abuso, pena, castigo); [agarrar, coger] (alguien) (a alguien) de [chancho, maje, mona] (DdAm, Costa Rica) ( burlarse una persona de alguien);

En el segundo caso tendríamos variaciones del tipo gastar alguien pólvora en [chimangos, gallinazo, zamuros, zanate] (DRAE; hacer esfuerzos por algo o alguien que, en realidad, tiene poca importancia); cagar alguien fuera del [cajón, tibor].

El diccionario de la RAE indica (ver captura de pantalla $\mathrm{n}^{\circ} 1$ ) gastar alguien pólvora en chimangos se .usa en Arg; Par y Ur. gastar alguien pólvora en gallinazo se usa en Ec. gastar alguien pólvora en zamuros se usa en Ven. gastar alguien pólvora en zanates se usa en El Salv.

En cuanto a polisemia por ahora tenemos ya casi 9000 casos de polisemia de los que 2.800 corresponden a CVF diatópicas. Por ej: caerse alguien de la cama:

1. (DRAE; Cuba) (Ser demasiado crédulo);

2. (DdAm; EE:UU; Pa, Cu, Ven) (Enfermar).

\section{Conclusión}

La investigación desarrollada por los integrantes del grupo FRASYTRAM para elaborar una gran base de datos de construcciones verbales multilingüe, clasificadas semánticamente y con equivalentes en varios idiomas, nos ha permitido comprobar que 
existe una enorme riqueza fraseológica en español tanto en el caso del español peninsular como en el de las variantes diatópicas del español hablado en Hispanoamérica. Cada uno de los países hispanoamericanos que usa el español como lengua oficial ha creado centenares, y posiblemente miles de UF idiosincrásicas que reflejan la peculiaridad de su sociedad y cultura a través de creaciones lingüísticas. Sin embargo, esa riqueza fraseológica diatópica no se ve reflejada en los contenidos fraseológicos de los diccionarios planteando los consiguientes problemas de comprensión y de traducción a los usuarios del español. Existe un desfase considerable entre el continuo e imparable proceso de remodelación de los actos de comunicación constantemente obligados a readaptarse y el material que los lexicógrafos insertan en los diccionarios. En efecto, el análisis de la BD que está recopilando las CVF, muestra que los diccionarios españoles incorporan un muy escaso número de CVF diatópicas. De 20.600 CVF presentes en diccionarios españoles, solamente un $0.5 \%$ corresponde a CVF diatópicas hispanoamericanas. Sin embargo existen miles y miles de UF diatópicas hispanoamericanas y la importancia numérica de la población mundial que habla español aumenta considerablemente en esos países así como el peso de su economía dando un vuelco a la importancia de las variedades del español por lo que convendría incorporar un número más importante de expresiones usuales en ese continente. Por otro lado, los diccionarios hispanoamericanos incluyen solamente creaciones usuales en sus países dejando de lado otras expresiones que pertenecerían a un español común por lo que a menudo no se sabe si esas UF que pertenecen al español estándar son conocidas y usadas por los hablantes hispanoamericanos. Convendría pues realizar un estudio riguroso que permitiese elaborar un núcleo de UF usuales comunes a la mayoría de los países que utilizan el español a diario como lengua oficial en la óptica de los trabajos realizados en la francofonía con el proyecto BFQS (Ladmiroy 2010).

Universidad de Alicante

\section{Bibliografía}

Corpas Pastor, G. 1996. Manual de fraseología española. Madrid: Gredos. García-Page, M. 2008. Introducción a la fraseología española. Barcelona: Estudio de locuciones. Anthropos.

Lamiroy B. 2010. Les expresssions verbales figées de la francophonie. Gap-Paris: Ophrys. Mogorrón Huerta, P. 2004. Los diccionarios electrónicos fraseológicos, perspectivas para la lengua y la traducción. E.L.U.A.. 12. 381-400.

Mogorrón Huerta, P. 2008. Traduction et compréhension des locutions verbales. Meta 53 (2). 378-406.

Mogorrón Huerta, P. 2008. Diccionario electrónico multilingüe de construcciones verbales aplicado a la traducción. In Mellado Blanco, C (ed), Colocaciones y fraseología en los diccionarios. 263-281. Frankfurt: Peter Lang. 
Mogorrón Huerta, P \& Mejri,S (eds). 2010. Opacidad, idiomaticidad y traducción. Quinta impresión. Universidad de Alicante.

Mogorrón Huerta, P. 2010. La Opacidad en las construcciones verbales fijas. In Mogorrón Huerta, P \& Mejri,S (eds). 2010. Opacidad, idiomaticidad y traducción, 236-260. Quinta impresión.Universidad de Alicante.

Mogorrón Huerta, P. 2010. Analyse du figement et de ses possibles variations dans les constructions verbales espagnoles. Linguisticae Investigationes 33 (1). 86-153.

Mogorrón Huerta, P. 2010. Estudio contrastivo lingüístico y semántico de las construcciones verbales fijas diatópicas mexicanas/españolas. Quaderns de Filologia. Estudis lingüístics 15. 179-198.

Mogorrón Huerta, P. 2010. Las construcciones verbales fijas con contenidos somáticos en los diccionarios españoles. In De Miguel J.C, Hernández C, Pinilla, J (eds), Enfoques de Teoría, Traducción y didáctica de la lengua francesa.249-261. Universitat de València.

Mogorrón Huerta, P 2011. Compétence phraséologique et traitement des expressions figées dans les dictionnaires. In Passeurs de mots, passeurs d'espoir. Lexicologie, terminologie et traduction face au défi de la diversité. Agence Universitaire pour la Francophonie. 517-535.

Ruiz Gurillo, L. 1997. Aspectos de fraseología teórica y aplicada, Universidad de Valencia, anejo 24 de $C F$.

Zuluaga, A. 1980. Introducción al estudio de las expresiones fijas. Frankfurt : Studia Romancia, $\mathrm{n}^{\circ} 10$, Verlag Peter Lang.

\section{Dicccionarios}

AoMex = Diccionario breve de mexicanismos. http://www.academia.org.mx/diccionarios/

DICAZ/inicio.htm.

DDA = Diccionario del español de Argentina. Español de Argentina - Español de España, Madrid: Gredos.2000.

DDAM= Diccionario de Americanismos.Santillana. 2010.

DEA = Diccionario del español Actual .Madrid: Aguilar Lexicografía.2005.

DEBI = Diccionario Espasa bilingüe Español-Francés/Francés-español. Espasa. 2000.

DEREC $=$ Diccionario de Expresiones y Refranes del español de Canarias . Cabildo de Gran Canaria. 2000.

DEUEM = Diccionario del español usual en México (1996). México: El colegio de México.. http:// www.cervantesvirtual.com/servlet/SirveObras/35716130101359941976613/index.htm.

DEUEM = Diccionario del español usual en México.. El colegio de México. 2009.

DFDEM = Diccionario fraseológico del español moderno. Madrid: Santillana Ediciones Generales. 2005.

DFDHA = Diccionario fraseológico del habla argentina. María Gabriela Pauer|Pedro Luis Barcia. 2010.

DFDEA = Diccionario fraseológico documentado del español actual. Madrid: Aguilar Lexicografía.2004.

DHDA = Diccionario del habla de los argentinos, Academia Argentina de Letras, Buenos Aires: Espasa Calpe. 2003.

DRAE = Diccionario de la Real Academia Española. Madrid: Espasa Calpe. 2001. 
DT = Diccionario temático de locuciones francesas con su correspondencia española. Madrid: Gredos. 2004.

DTDFH = Diccionario temático de frases hechas. Barcelona: Columbus. 2004.

DM = Diccionario de Mexicanismos, Academia Mexicana de la lengua, Siglo XXI, 2010.

DUE = Diccionario de uso del español. Madrid: Gredos. 1999.

$\mathrm{EBI}=$ Diccionario Espasa Gran Español-Francés / Francés-español. Espasa. Madrid.2000.

$\mathrm{EPM}=$ Enciclopedia Planeta multimedia- DVD-ROM. 2003.

LBI = Gran diccionario Larousse Español-Francés/Francés-español. Larousse. Barcelona. 1999. LIBSA = Jergas, Argot y Modismos .Madrid. LIBSA. 2001.

NDEA = Nuevo Diccionario Etimológico Aragonés. Gara d’Edizions, 2000.

SL = LAFAGE, Suzanne (1997-1998) : Lexique du Français de Côte d'Ivoire (Appropriation et créativité). 